\title{
Serum Lactate/Albumin as a Predictor of In- Hospital Mortality in ICU Pancreatitis Patients: an Analysis of the MIMIC-III Database
}

\section{Peng Bao}

Affiliated Hospital of Nantong University

\section{Xiaoli Cui}

Affiliated Hospital of Nantong University

Haoliang Shen

Affiliated Hospital of Nantong University

Yiping Wang

Affiliated Hospital of Nantong University

Yang Lu ( $\nabla$ marineluyang@ntu.edu.cn )

Affiliated Hospital of Nantong University

\section{Research Article}

Keywords: Acute pancreatitis, Lactate, Albumin, Outcome, MIMIC III database

Posted Date: December 16th, 2021

DOI: https://doi.org/10.21203/rs.3.rs-1166460/v1

License: (c) (1) This work is licensed under a Creative Commons Attribution 4.0 International License. Read Full License 


\section{Abstract}

Background: Acute pancreatitis (AP) is a common serious illness, and is characterized by rapid deterioration and a high mortality rate. Several biomarkers can evaluate and guide the treatment of acute pancreatitis, but there is currently no consensus on which markers are the most effective, simple, and economical for treating early-onset AP. In this study, we used the MIMIC III database to conduct a retrospective study on the relationship between early lactate/albumin (LAC/ALB), in-hospital mortality, and complication rates in patients with acute pancreatitis in the ICU.

Methods: Basic data and indicators of laboratory tests, hospital deaths, and hospitalization days of acute pancreatitis patients were extracted from the database, after which the relationship between LAC/ALB and hospital mortality, ICU hospitalization days, and organ failure were evaluated using a $t$-test, a ranksum test, a chi-square test or Fisher's exact probability method, and a Cox proportional hazard model.

Results: 894 patients met the requirements and were selected from the MIMIC III database. They were subsequently grouped according to the lower limit ratio of the LAC/ALB normal value of 0.7 . The group with LAC/ALB>0.7 showed higher hospital mortality rates, and the Lac, Inr, nitrogen, blood sugar, AKI incidence, Tbil, Sapsii score, and Sofa scores were all higher than the group with $L A C / A L B<0.7$. A multivariate Cox regression analysis model was used to explore the relationship between LAC/ALB levels and inpatient mortality. After including different adjustment variables, we determined that LAC/ALB is a risk factor for in-hospital death. The results of the subgroup analysis of LAC/ALB levels and mortality of hospitalized patients indicate that higher levels of LAC/ALB are risk factors for in-hospital deaths in patients with acute pancreatitis.

\section{Background}

AP is a common serious illness characterized by rapid progression and high mortality, and can easily lead to multiple organ failure (MODS). The current treatment for acute pancreatitis primarily includes fasting, gastrointestinal decompression, acid-inhibiting pancreatin, fluid resuscitation, early and minimally invasive drainage, organ function support, and surgery ${ }^{[1]}$. If not effectively treated in its early stage, AP will often develop into severe acute pancreatitis (SAP). Pancreatitis patients admitted to the ICU have a poor prognosis and high mortality ${ }^{[2]}$. The Acute Physiology and Chronic Health Score (APACHE II) Ranson score is used to predict acute pancreatitis, however, its use in clinical practice is limited due to the difficult calculations and several parameters needed ${ }^{[3]}$. Therefore, it is particularly important to find fast, simple, and accurate predictive indicators.

Lac is an important indicator of organ tissue perfusion. During hypoxia and tissue hypoperfusion, lactate dehydrogenase will reduce the degradation of pyruvate, leading to the accumulation of lactic acid ${ }^{[4]}$. Previous studies have demonstrated that hyperlactic acidemia is closely related to prognostic outcomes in critically ill patients ${ }^{[5-7]}$. Additionally, elevated levels of lactate are an independent risk factor for poor prognosis in patients with pancreatitis ${ }^{[8]}$. However, using only lactic acid as a disease prognostic 
indicator produces unreliable results. For example, the blood lactic acid clearance rate in patients with liver dysfunction often decreases ${ }^{[9]}$. Serum albumin (ALB) is the main protein in human plasma and plays an important role in nutrition, anti-inflammatory, anti-oxidation, and anabolic metabolism in the human body. Hypoproteinemia is also associated with high mortality in ICU patients with sepsis ${ }^{[10]}$. Similarly, ALB is closely related to MODS occurrence in the pancreatitis model ${ }^{[11]}$. ALB levels are typically affected by different factors. Critically ill patients in the ICU are typically in a state of stress, and their poor nutritional status and negative nitrogen balances usually lead to low ALB levels. Early fluid resuscitation could also reduce ALB levels, meaning there could be errors if only ALB levels are used as a predictor. It has been reported that $L A C / A L B$ can effectively predict the in-hospital mortality and prognosis of patients with sepsis ${ }^{[12,13]}$ better than Lac and Alb alone. The higher the LAC/ALB ratio of early patients, the higher the possibility of MODS in sepsis patients ${ }^{[14]}$. Based on previous studies, LAC/ALB could be able to assess and predict the condition of patients with acute pancreatitis. Our goal is to use the LAC/ALB ratio to predict the in-hospital mortality rate and MODS of patients with acute pancreatitis.

\section{Methods}

Data source. Data were obtained from a public and free clinical data database, the Multiparameter Intelligent Monitoring in Intensive Care III (MIMIC III, V1.4), which includes 46,520 patients admitted to Beth Israel Deaconess Medical Center in Boston from 2001 to 2012 ${ }^{[15]}$. The database primarily records the basic information of hospitalized patients, including vital signs, laboratory tests, water intake and discharge, and treatment measures. The database diagnoses and classifies patients according to the International Classification of Diseases, Ninth Revision (ICD-9). We completed the National Institutes of Health's web-based course and passed the Protecting Human Research Participants exam(N0.37333883). Our research was approved by the Institutional Review Boards of Beth Israel Deaconess Medical Center and the Massachusetts Institute of Technology (Cambridge, MA).The study was reviewed by the Ethics Committee of the Affiliated Hospital of Nantong University, and no further ethics approval was required.

Population Selection Criteria. A total of 961 patients participated, all of whom were diagnosed with acute pancreatitis based on the ICD-9 code (icd9_code=5770). Based on their first hospitalization record, we excluded patients younger than 18 years old and hospitalized in the ICU for less than 24 hours. In the end, 894 patients were selected for this study.

Data extraction and management. PostgresSQL (version 9.6) was used to extract data from the MIMIC-III database. Basic patient information includes age, gender, vital signs, laboratory test results, scoring system, underlying diseases, and organ support methods. Underlying diseases primarily include cholelithiasis, chronic obstructive pulmonary disease (COPD), coronary heart disease, hypertension, and diabetes. In addition, laboratory test data were also extracted, including ALB, blood amylase, blood creatinine, hemoglobin, Lac, platelets, nitrogen, total bile, triglycerides, and glucose. Vital signs include 
heart rate, systolic blood pressure, diastolic blood pressure, pulse, respiratory rate, and body temperature. The scoring system primarily includes simplified acute physiological score (Sapsii) and continuous organ dysfunction score (SOFA). Other selected items include whether to use ventilator mechanical ventilation, whether to use hypertensive drugs, and whether it is complicated by acute kidney injury (AKI).

Statistical Analysis. The mean \pm standard deviation was used when continuous variables followed a normal distribution, otherwise, the median (minimum, maximum) was used; the categorical variable data is indicated by frequency (percentage). When comparing between groups, a $t$-test was used if continuous variables meet the normal distribution, and the rank-sum test was used if continuous variables do not follow a normal distribution. The chi-square test or Fisher's exact probability method was used for categorical variables. The KM method was used to draw the survival curve, and the log-rank test was used to compare whether the LAC/ALB stratification is statistically different. Cox proportional-hazards model was used to evaluate the relationship between LAC/ALB and hospital mortality, with LAC/ALB<0.7 serving as the reference group. In the crude model, no covariates were included; Model I was adjusted by age and gender; Model $\varangle$ was based on model I and also incorporated laboratory tests, vital signs, Sofa, and Sapsii scores, whether to use blood pressure drugs, concurrent AKI, and combined underlying diseases as covariates. The regression results of the Cox proportional-hazards model are expressed by an HR value and a 95\% confidence interval. In the subgroup analysis, a hierarchical exploration was performed for each index to identify interactions between LAC/ALB and each index. All statistical analyses were performed with Stata 16.0 software. The hypothesis test was performed on both sides, with $p<0.05$ representing a significant statistical difference.

\section{Results}

\section{Baseline characteristics and outcomes}

In this study, we observed and summarized the baseline characteristics, vital signs, laboratory test results, organ function, and prognosis of 894 patients (Figure1 and Table 1). The patients were divided into two groups according to their LAC/ALB levels: groups $<0.7$ and $\geq 0.7$ groups. Of the patients, 499 were males and 395 were females with average ages of $63.81 \pm 1.86$ years old and $68.40 \pm 2.34$ years old, respectively. There were no significant differences between gender and age groups. The levels of Lac, Inr, Nitrogen, Tbil, and Glucose and the scores of Sapsii and Sofa in the LAC/ALB $\geq 0.7$ group were both higher than in the $<0.7$ group. Compared with the LAC/ALB $<0.7$ group, patients in the LAC/ALB $\geq 0.7$ group were more likely to have AKI during hospitalization $(22.25 \% \mathrm{VS} 15.63 \%, p=0.011)$, while the hospital mortality rate was also significantly higher $(23.65 \%$ VS8 $.14 \%, p<0.001)$. However, there was no significant difference in ICU hospitalization time between the two groups.

\section{High LAC/ALB ratio is an independent prognostic predictor in pancreatitis patients}


As shown in Figure 2, the influence of the LAC/ALB ratio on the prognosis of patients with acute pancreatitis was explored by analyzing the survival rate. The median survival and $95 \mathrm{Cl}$ in the LAC/ALB<0.7 group and $\geq 0.7$ group were $80(50,95)$ days and $56(47,67)$ days, respectively, indicating that patients with high LAC/ALB ratios have higher mortality. Additionally, we also performed a multivariate Cox regression analysis on the baseline variables (Table 2). We analyzed LAC/ALB levels using continuous variables and categorical variables (classified based on whether it was less than 0.7) and explored the relationship between LAC/ALB levels and the mortality rate of hospitalized patients. In the crude model, no adjustment variable was added, and the continuous variable results demonstrated that the HR and its $95 \% \mathrm{Cl}$ value were $[2.181(1.935,2.457)]$. For categorical variables, the LAC/ALB<0.7 group was used as a reference, while the HR and $95 \% \mathrm{Cl}$ value of the LAC/ALB $\geq 0.7$ group is [2.641 $(1.808,3.857)]$. We adjusted model I by age and gender and the result was the same as in the crude model. The continuous variable results demonstrated that $\mathrm{HR}$ and its $95 \% \mathrm{Cl}$ value was [2.285 (2.021, 2.584)]. For the categorical variable, the $<0.7$ group was used as a reference, while the $\mathrm{HR}$ and its $95 \% \mathrm{Cl}$ value in the $\geq 0.7$ group is $[2.684(1.838,3.920)]$. We adjusted model II based on model I by including Tbil, Plates, Amy, Nitrogen, Triglycerides, Hbg,

Hematocrit, Creatinine, Glucose, Sysbp, Diasbp, Pulsrate, Resprate, Temperature, SpO2, Sofa, Sapsii, Inr, Ventdurate, Hyperensort use, and AKI. The continuous variable results demonstrated that its $\mathrm{HR}$ and its $95 \% \mathrm{Cl}$ value was [2.016 $(1.700,2.390)]$, while the categorical variables results demonstrated that the $\mathrm{HR}$ and its $95 \% \mathrm{Cl}$ value of $\mathrm{LAC} / \mathrm{ALB} \geq 0.7$ group were $[1.575(1.026,2.416)]$. These all indicate that higher LAC/ALB levels are risk factors for death in hospitalized patients.

\section{Subgroup analysis}

The subgroup analysis of LAC/ALB levels and the mortality of hospitalized patients are shown in Table 3. No interactions were observed in each stratification $(p=0.212 \sim 0.911)$. The results from the subgroups of Tbil, INR, Platelet, Amy, Hbg, Creatinine, Glucose, SBP, DBP, Heart rate, Respiratory rate, Temperature, SPO2, SOFA, SAPSII, and Ventdurate all demonstrated that higher levels of LAC/ALB are risk factors for death in hospitalized patients $(p<0.05)$.

\section{Discussion}

AP is characterized by severe symptoms, rapid clinical progression, and high mortality rates. If effective treatment is not available in the early stages, MODS will be particularly acute in the severe AP stage ${ }^{[16]}$. It is particularly important to identify indicators that can assess the severity of AP patients during the early stages. Current early-identification methods for high-risk patients are high-resolution ${ }^{[17]}$ and modified Marshall scoring systems ${ }^{[18]}$, but a positive result is typically not noticed before the disease progresses. According to current research, C-reactive protein (CRP) ${ }^{[19]}$, procalcitonin (PCT) ${ }^{[20]}$, changes in serum lactic acid levels ${ }^{[21]}$, and the product value of serum albumin and prothrombin ${ }^{[22]}$ have all been used as predictors of AP severity. However, the sample size of previous studies was small, or using a single indicator was inaccurate. Therefore, we need to find other fast, accurate, and cheap methods of 
identifying AP in its early stages. The results of our study assessing 894 AP patients supplies additional evidence.

Lac and Alb are economical and convenient laboratory indicators that are easy to clinically obtain. However, their individual use is complicated by many clinical factors. The upper limit of the normal value of Lac is $2.5 \mathrm{mmol} / \mathrm{L}$, and the lower limit of the normal value of Alb is $3.5 \mathrm{mg} / \mathrm{L}$. This ratio is used for grouping. Our results demonstrated that there is no statistical difference in age and gender between the two groups ( $p=0.1227, p=0.964)$. Based on the $t$-test results, there were significant differences between the two groups in INR and platelet count $(p=0.0122, p<0.0001)$. Previous studies confirmed that the increased vascular permeability of SAP patients formed an embolization of the blood vessels between the pancreatic acinars, and initiated the exogenous coagulation pathway through the tissue factor pathway, while platelet aggregation initiated the endogenous coagulation pathway by the overactivation of two coagulation pathways, eventually leading to hyperfibrinolysis ${ }^{[23]}$. The decline of platelets could be caused by the significant accumulation of PLT due to endothelial damage and the destruction of platelets by a large number of inflammatory factors in the blood circulation. Abdominal hemorrhage in patients with pancreatitis caused by coagulation dysfunction is another primary cause of death ${ }^{[24]}$. We found that patients with LAC/ALB $>0.7$ had higher blood glucose levels. When SAP attacks, pancreatic islet cells are destroyed and insulin secretion is reduced. Stress factors such as hypotension and shock can cause the excessive pancreatic release of glucose-increasing hormones such as glucagon and glucocorticoids. This can also lead to increased blood glucose stress. For example, during the early study of diabetes, it was generally believed that the greater the blood glucose fluctuations, the more serious the pancreatic exudation and necrosis, which would lead to higher mortality rates. However, recent clinical studies have demonstrated that when the body's blood sugar control is poor, the risk of infection increases, inflammatory mediators are released, and pancreatic infection and exudation increase, which aggravates severe AP patients and significantly increases patient mortality ${ }^{[25]}$. The level of urea nitrogen was significantly different between the two groups $(p=0.0027)$. As a common indicator for evaluating kidney function, higher urea nitrogen levels are related to peripancreatic exudation and necrosis. The level of urea nitrogen is largely affected by changes in blood vessel volume; therefore, levels of urea nitrogen upon admission could indicate that the patient has potential intravascular volume exhaustion and prerenal azotemia, increasing the risk of complications and mortality if it is not treated early ${ }^{[26]}$. Our research results confirmed that patients with elevated urea nitrogen are more likely to develop AKI. Additionally, patients in the LAC/ALB group $>0.7$ have fast heart rates, low blood pressure, and high breathing rate, all of which could be related to SIRS as the condition worsens. The ability of the SOFA score and SAPII score to evaluate disease severity has been confirmed in sepsis and pancreatitis patients [27]. In our study, we found that symptoms in patients with hyperlactic acid and hypoproteinemia were more serious. Our results were subjected to a Chi-square test, $t$-test, Cox proportional hazard model, and subgroup analysis have effectively confirmed that LAC/ALB $>0.7$ is an independent risk factor for inhospital death in ICU patients with AP. 
Our research indicates that the hospital mortality rate of an AP patient admitted to the ICU will be reduced if special attention is paid to serum lactic acid levels during early fluid resuscitation, the perfusion of organs and tissues, and maintaining a suitable blood pressure level. Hypoalbuminemia should be paid special attention during fluid resuscitation. Our follow-up studies will further confirm whether active albumin infusion and enhanced nutritional support will reduce the mortality of patients with pancreatitis.

This study has several limitations. First, patients selected for a retrospective study could be biased. For example, AP of pancreatitis according to the ICD_9 code might not be the primary reason for their hospital admission. Second, the database in this study is a single-center study, and additional cases should be considered from other medical centers to confirm our results. Third, there is a lack of follow-up on patient outcomes.

\section{Conclusion}

Pancreatitis progresses rapidly and mortality is higher in patients admitted to ICU. In this study, early $\mathrm{Lac} /$ Alb was found to be an independent risk factor for pancreatitis in ICU, suggesting that attention should be paid to serum albumin levels during early fluid resuscitation.

\section{Declarations}

\section{Acknowledgements}

Not applicable.

\section{Authors' contributions}

BP collected the data, designed the study, and wrote the paper. SHL and WYP followed the study, and wrote the paper. CXL collected the data. LY followed the study and critically revised the paper. All authors read and approved the final manuscript.

\section{Funding}

This study was supported by the Nantong Science and Technology Bureau of Jiangsu Province 2020 Mandatory project(NO.XNBH00031800).

\section{Availability of data and materials}

The data set supporting the results of this article are included within the article.

\section{Ethics approval and consent to participate}

Data were obtained from a public and free clinical data database, the Multiparameter Intelligent Monitoring in Intensive Care III (MIMIC III, V1.4). We completed the National Institutes of Health's webbased course and passed the Protecting Human Research Participants exam(NO.37333883). Our 
research was approved by the Institutional Review Boards of Beth Israel Deaconess Medical Center and the Massachusetts Institute of Technology (Cambridge, MA). The study was reviewed by the Ethics Committee of the

Affiliated Hospital of Nantong University, and no further ethics approval was required.

Not applicable.

\section{Competing interests}

The authors read and approved the final manuscript.

\section{Author details}

${ }^{1}$ The department of intensive care unit, Affiliated Hospital of Nantong University

\section{References}

1. Huber W, Algül H. [Treatment of acute necrotizing pancreatitis]. Internist (Berl). 2019. 60(3): 226-234.

2. Jiang $X$, Su Z, Wang $Y$, et al. Prognostic nomogram for acute pancreatitis patients: An analysis of publicly electronic healthcare records in intensive care unit. J Crit Care. 2019. 50: 213-220.

3. Rau B, Bothe A, Beger HG. Surgical treatment of necrotizing pancreatitis by necrosectomy and closed lavage: changing patient characteristics and outcome in a 19-year, single-center series. Surgery. 2005. 138(1): 28-39.

4. Samaraweera SA, Gibbons B, Gour A, Sedgwick P. Arterial versus venous lactate: a measure of sepsis in children. Eur J Pediatr. 2017. 176(8): 1055-1060.

5. BRODER G, WEIL MH. EXCESS LACTATE: AN INDEX OF REVERSIBILITY OF SHOCK IN HUMAN PATIENTS. Science. 1964. 143(3613): 1457-9.

6. Vincent JL, Dufaye P, Berré J, Leeman M, Degaute JP, Kahn RJ. Serial lactate determinations during circulatory shock. Crit Care Med. 1983. 11(6): 449-51.

7. Andersen LW, Mackenhauer J, Roberts JC, Berg KM, Cocchi MN, Donnino MW. Etiology and therapeutic approach to elevated lactate levels. Mayo Clin Proc. 2013. 88(10): 1127-40.

8. Zhang W, Zhang M, Kuang Z, Huang Z, Gao L, Zhu J. The risk factors for acute respiratory distress syndrome in patients with severe acute pancreatitis: A retrospective analysis. Medicine (Baltimore). 2021. 100(2): e23982.

9. Jeppesen JB, Mortensen C, Bendtsen F, Møller S. Lactate metabolism in chronic liver disease. Scand J Clin Lab Invest. 2013. 73(4): 293-9.

10. Takegawa R, Kabata D, Shimizu K, et al. Serum albumin as a risk factor for death in patients with prolonged sepsis: An observational study. J Crit Care. 2019. 51: 139-144. 
11. Li S, Zhang Y, Li M, Xie C, Wu H. Serum albumin, a good indicator of persistent organ failure in acute pancreatitis. BMC Gastroenterol. 2017. 17(1): 59.

12. Shin J, Hwang SY, Jo IJ, et al. Prognostic Value of The Lactate/Albumin Ratio for Predicting 28-Day Mortality in Critically ILL Sepsis Patients. Shock. 2018. 50(5): 545-550.

13. Kong T, Chung SP, Lee HS, et al. The Prognostic Usefulness of the Lactate/Albumin Ratio for Predicting Clinical Outcomes in Out-of-Hospital Cardiac Arrest: a Prospective, Multicenter Observational Study (koCARC) Study. Shock. 2020. 53(4): 442-451.

14. Gharipour A, Razavi R, Gharipour M, Mukasa D. Lactate/albumin ratio: An early prognostic marker in critically ill patients. Am J Emerg Med. 2020. 38(10): 2088-2095.

15. Johnson AE, Pollard TJ, Shen L, et al. MIMIC-III, a freely accessible critical care database. Sci Data. 2016. 3: 160035.

16. Ge P, Luo Y, Okoye CS, et al. Intestinal barrier damage, systemic inflammatory response syndrome, and acute lung injury: A troublesome trio for acute pancreatitis. Biomed Pharmacother. 2020. 132: 110770.

17. Zhao K, Adam SZ, Keswani RN, Horowitz JM, Miller FH. Acute Pancreatitis: Revised Atlanta Classification and the Role of Cross-Sectional Imaging. AJR Am J Roentgenol. 2015. 205(1): W32-41.

18. Banks PA, Bollen TL, Dervenis $C$, et al. Classification of acute pancreatitis--2012: revision of the Atlanta classification and definitions by international consensus. Gut. 2013. 62(1): 102-11.

19. Riché FC, Cholley BP, Laisné MJ, et al. Inflammatory cytokines, $C$ reactive protein, and procalcitonin as early predictors of necrosis infection in acute necrotizing pancreatitis. Surgery. 2003. 133(3): 25762.

20. Müller CA, Uhl W, Printzen G, et al. Role of procalcitonin and granulocyte colony stimulating factor in the early prediction of infected necrosis in severe acute pancreatitis. Gut. 2000. 46(2): 233-8.

21. Shu W, Wan J, Chen J, et al. Elevated arterial lactate level as an independent risk factor for pancreatic infection in moderately severe acute pancreatitis. Pancreatology. 2019. 19(5): 653-657.

22. Li S, Liu Z, Wu H. The product value of serum albumin and prothrombin time activity could be a useful biomarker for severity prediction in AP: An ordinal retrospective study. Pancreatology. 2019. 19(2): 230-236.

23. Hegyi P, Pandol S, Venglovecz V, Rakonczay Z Jr. The acinar-ductal tango in the pathogenesis of acute pancreatitis. Gut. 2011. 60(4): 544-52.

24. Tauchi R, Imagama S, Ito Z, et al. Acute pancreatitis after spine surgery: a case report and review of literature. Eur J Orthop Surg Traumatol. 2014. 24 Suppl 1: S305-9.

25. Kikuta K, Masamune A, Shimosegawa T. Impaired glucose tolerance in acute pancreatitis. World J Gastroenterol. 2015. 21(24): 7367-74.

26. Bollen TL, Singh VK, Maurer R, et al. A comparative evaluation of radiologic and clinical scoring systems in the early prediction of severity in acute pancreatitis. Am J Gastroenterol. 2012. 107(4): 612-9. 
27. Biberci Keskin E, Taşlıdere B, Koçhan K, Gülen B, İnce AT, Şentürk H. Comparison of scoring systems used in acute pancreatitis for predicting major adverse events. Gastroenterol Hepatol. 2020. 43(4): 193-199.

\section{Tables}

Table 1 Baseline characteristics, vital signs, laboratory parameters and outcomes of patients with Acute pancreatitis 


\begin{tabular}{|c|c|c|c|c|}
\hline \multirow{3}{*}{ Characteristics } & \multicolumn{4}{|l|}{ LAC_ALB } \\
\hline & $<0.7$ & $\geq 0.7$ & $t / Z / c^{2}$ value & $P$ value \\
\hline & $(n=467)$ & $(n=427)$ & & \\
\hline Age, years & $63.81 \pm 1.86$ & $68.40 \pm 2.34$ & 1.5450 & 0.1227 \\
\hline \multicolumn{5}{|l|}{ Gender, n(\%) } \\
\hline Female & $206(44.11)$ & $189(44.26)$ & 0.0021 & 0.964 \\
\hline Male & $261(55.89)$ & $238(55.74)$ & & \\
\hline \multicolumn{5}{|l|}{ Comorbidities } \\
\hline \multicolumn{5}{|l|}{ Diabetes, n(\%) } \\
\hline Yes & $119(25.48)$ & $105(24.59)$ & 0.0944 & 0.75 \\
\hline No & $348(74.52)$ & $322(75.41)$ & & \\
\hline \multicolumn{5}{|l|}{ Cholecystitis, n(\%) } \\
\hline Yes & $83(17.77)$ & $74(17.33)$ & 0.0302 & 0.862 \\
\hline No & $384(82.33)$ & $353(82.67)$ & & \\
\hline \multicolumn{5}{|l|}{ COPD, n(\%) } \\
\hline Yes & $51(10.92)$ & $30(7.03)$ & 4.1070 & 0.043 \\
\hline No & $416(89.08)$ & $397(92.97)$ & & \\
\hline \multicolumn{5}{|l|}{ Coronary, n(\%) } \\
\hline Yes & $82(17.56)$ & 78 (18.27) & 0.0761 & 0.783 \\
\hline No & $385(82.44)$ & $349(91.73)$ & & \\
\hline \multicolumn{5}{|l|}{ Hypertension, n(\%) } \\
\hline Yes & $334(49.48)$ & $100(45.66)$ & 0.9657 & 0.326 \\
\hline No & $341(50.52)$ & $119(54.34)$ & & \\
\hline \multicolumn{5}{|c|}{ Laboratory parametes } \\
\hline $\operatorname{Alb}(g / d L)$ & $3.14 \pm 0.02$ & $2.83 \pm 0.30$ & 7.7105 & $<0.0001$ \\
\hline Amy(IU/L) & $225.10 \pm 12.22$ & $218.39 \pm 11.08$ & 0.4048 & 0.6857 \\
\hline Creatinine $(\mathrm{mg} / \mathrm{dL})$ & $1.51 \pm 0.07$ & $1.60 \pm 0.07$ & 0.8786 & 0.3799 \\
\hline $\mathrm{Hbg}(\mathrm{g} / \mathrm{dL})$ & $10.32 \pm 0.12$ & $10.34 \pm 0.14$ & 0.0974 & 0.9225 \\
\hline Hematocrit( \%) & $31.07 \pm 0.18$ & $31.16 \pm 0.19$ & 0.3347 & 0.7379 \\
\hline
\end{tabular}




\begin{tabular}{|c|c|c|c|c|}
\hline $\mathrm{Lac}(\mathrm{mmol} / \mathrm{L})$ & $1.46 \pm 0.02$ & $3.68 \pm 0.15$ & 17091 & $<0.0001$ \\
\hline Lac_Alb_ratio & $0.47 \pm 0.01$ & $1.38 \pm 0.05$ & 20.5034 & $<0.0001$ \\
\hline $\ln r$ & $1.38 \pm 0.02$ & $1.65 \pm 0.11$ & 2.5100 & 0.0122 \\
\hline Nitrogen $(\mathrm{mg} / \mathrm{dL})$ & $25.78 \pm 0.87$ & $29.74 \pm 1.00$ & 3.0102 & 0.0027 \\
\hline Plates & $291.53 \pm 6.52$ & $248.09 \pm 6.84$ & 4.5973 & $<0.0001$ \\
\hline Tbil(mg/dL) & $1.56 \pm 0.15$ & $3.05 \pm 0.23$ & 5.5334 & $<0.0001$ \\
\hline Triglycerides $(\mathrm{mg} / \mathrm{dL})$ & $206.57 \pm 14.81$ & $228.40 \pm 20.88$ & 0.8760 & 0.3814 \\
\hline Glucose(mmol/L) & $135.23 \pm 1.74$ & $142.87 \pm 2.26$ & 2.7073 & 0.0069 \\
\hline \multicolumn{5}{|l|}{ Vital sighs } \\
\hline Heartrate, bpm & $92.62 \pm 0.78$ & $95.00 \pm 0.84$ & 2.0927 & 0.0367 \\
\hline Sysbp, mmHg & $125.03 \pm 0.82$ & $119.38 \pm 0.86$ & 4.7568 & $<0.0001$ \\
\hline Diasbp, mmHg & $65.12 \pm 0.57$ & $63.45 \pm 0.60$ & 2.2095 & 0.0427 \\
\hline Pulsrate, bpm & $82.19 \pm 0.56$ & $80.10 \pm 0.58$ & 2.5748 & 0.0102 \\
\hline Resprate, bmp & $20.29 \pm 0.20$ & $20.87 \pm 0.21$ & 2.0354 & 0.0421 \\
\hline Temperature, ${ }^{\circ} \mathrm{C}$ & $37.11 \pm 0.03$ & $36.93 \pm 0.03$ & 4.1225 & $<0.0001$ \\
\hline $\mathrm{SpO2}$ & $96.78 \pm 0.08$ & $96.46 \pm 0.16$ & 1.8283 & 0.0678 \\
\hline \multicolumn{5}{|l|}{ Score system } \\
\hline Sofa scores & $4.44 \pm 0.14$ & $6.02 \pm 0.21$ & 6.3966 & $<0.0001$ \\
\hline Sapsii scores & $32.75 \pm 0.59$ & $41.06 \pm 0.88$ & 7.9714 & $<0.0001$ \\
\hline \multicolumn{5}{|l|}{ Outcome } \\
\hline ICUday, days & $3(1,97)$ & $3(179)$ & 0.346 & 0.7295 \\
\hline \multicolumn{5}{|l|}{ Hospital_expire, n(\%) } \\
\hline Yes & $38(8.14)$ & $101(23.65)$ & 40.8978 & $<0.001$ \\
\hline No & $429(91.86)$ & $326(76.35)$ & & \\
\hline \multicolumn{5}{|l|}{ Hypertension, n(\%) } \\
\hline Yes & $244(52.25)$ & $190(44.50)$ & 5.3660 & 0.021 \\
\hline No & $223(47.75)$ & $237(55.50)$ & & \\
\hline \multicolumn{5}{|l|}{$A K I, n(\%)$} \\
\hline Yes & $73(15.63)$ & $95(22.25)$ & 6.3988 & 0.011 \\
\hline
\end{tabular}




\begin{tabular}{|lllll|} 
No & $394(84.37)$ & $332(77.75)$ & & \\
\hline Hyperensort use, $\mathrm{n}(\%)$ & & & & \\
\hline Yes & $220(47.11)$ & $224(52.46)$ & 2.5537 & \\
No & $247(52.89)$ & $203(47.54)$ & & \\
Ventdurate, $\mathrm{n}(\%)$ & & & & \\
Yes & $220(47.11)$ & $224(52.46)$ & 2.5537 & \\
\hline No & $247(52.89)$ & $203(47.54)$ & & \\
\hline
\end{tabular}

Table 2 Relationship between LAC/ALB and hospital expired in different models.

\begin{tabular}{|c|c|c|c|c|c|c|}
\hline \multirow[t]{2}{*}{ Variable } & \multicolumn{2}{|l|}{ Crude model } & \multicolumn{2}{|l|}{ Model I } & \multicolumn{2}{|l|}{ Model II } \\
\hline & HR (95\% Cls) & $\begin{array}{l}P \\
\text { value }\end{array}$ & HR (95\% Cls) & $\begin{array}{l}P \\
\text { value }\end{array}$ & HR (95\% Cls) & $\begin{array}{l}P \\
\text { value }\end{array}$ \\
\hline \multicolumn{7}{|c|}{ Hospital expired } \\
\hline LAC/ALB \& & $\begin{array}{l}2.181(1.935 \\
2.457)\end{array}$ & $<0.001$ & $\begin{array}{l}2.285(2.021, \\
2.584)\end{array}$ & $<0.001$ & $\begin{array}{l}2.016(1.700 \\
2.390)\end{array}$ & $<0.001$ \\
\hline \multicolumn{7}{|c|}{$\begin{array}{l}\text { LAC/ALB } \\
\text { (Dichotomous) }\end{array}$} \\
\hline$<0.7$ & 1.0 (ref) & - & 1.0 (ref) & - & 1.0 (ref) & - \\
\hline$\geq 0.7$ & $\begin{array}{l}2.641(1.808 \\
3.857)\end{array}$ & $<0.001$ & $\begin{array}{l}2.684(1.838 \\
3.920)\end{array}$ & $<0.001$ & $\begin{array}{l}1.601(1.042 \\
2.458)\end{array}$ & 0.032 \\
\hline
\end{tabular}

HR: hazard ratio; $\mathrm{Cl}$ : confidence interval. Models were derived from Cox proportional hazards regression models. Crude model adjusted for: none. Model I adjusted for :age and gender.Model II adjusted for:Tbil[ Plates $\square$ Amy $\square$ Nitrogen $\square$ Triglycerides $\square \mathrm{Hbg} \square$ Hematocrit $\square$ Creatinine $\square$ Glucose $\llbracket$ Sysbp $\square$ Diasbp $\square$ Pulsrate $\square$

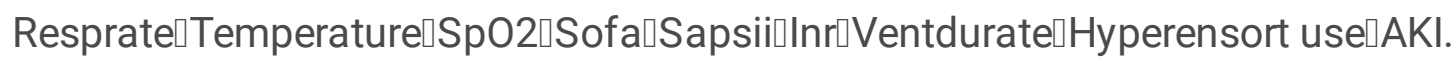

Table 3 Subgroup analysis of the associations between LAC/ALB ratio and hospital expire. 


\begin{tabular}{|c|c|c|c|c|}
\hline Characteristic & No.of patients & $\mathrm{HR}(95 \% \mathrm{Cls})$ & $P$ value & $P$ for interaction \\
\hline Total bilrubin (mg/dL) & & & & 0.700 \\
\hline$<1.0$ & 468 & $2.415(1.248,4.677)$ & 0.009 & \\
\hline$\geq 1.0$ & 423 & 2.196 (1.374 3.510) & 0.001 & \\
\hline INR & & & & 0.878 \\
\hline$<1.4$ & 560 & $2.247(1.289,3.915)$ & 0.004 & \\
\hline$\geq 1.4$ & 331 & $2.321(1.338,4.024)$ & 0.003 & \\
\hline Platelet $\left(10^{9} / \mathrm{L}\right)$ & & & & 0.664 \\
\hline$<226$ & 368 & $2.587(1.553,4.308)$ & $<0.001$ & \\
\hline$\geq 226$ & 523 & $2.010(1.121,3.605)$ & 0.019 & \\
\hline Amy & & & & 0.911 \\
\hline$<100$ & 251 & $2.854(1.199,6.795)$ & 0.018 & \\
\hline$\geq 100$ & 640 & $2.599(1.706,3.962)$ & $<0.001$ & \\
\hline Nitrogen & & & & 0.529 \\
\hline$<21$ & 430 & $2.152(0.891,5.198)$ & 0.088 & \\
\hline$\geq 21$ & 461 & $2.563(1.672,3.930)$ & $<0.001$ & \\
\hline Triglycerides & & & & 0.193 \\
\hline$<150$ & 317 & $1.598(0.834,3.062)$ & 0.158 & \\
\hline$\geq 150$ & 574 & $3.257(2.035,5.215)$ & $<0.001$ & \\
\hline $\mathrm{Hbg}(\mathrm{g} / \mathrm{dl})$ & & & & 0.762 \\
\hline$<12$ & 374 & $2.885(1.628,5.112)$ & $<0.001$ & \\
\hline$\geq 12$ & 517 & $2.600(1.557,4.340)$ & $<0.001$ & \\
\hline Hematocrit (\%) & & & & / \\
\hline$<40.0$ & 870 & $2.501(1.708,2.663)$ & $<0.001$ & \\
\hline$\geq 40.0$ & 21 & / & / & \\
\hline Creatinine (mg/dL) & & & & 0.521 \\
\hline$<1.4$ & 608 & $2.106(1.208,3.671)$ & $<0.001$ & \\
\hline$\geq 1.4$ & 283 & $2.758(1.626,4.677)$ & $<0.001$ & \\
\hline Glucose (mg/dL) & & & & 0.371 \\
\hline
\end{tabular}




\begin{tabular}{|c|c|c|c|}
\hline$<134$ & 462 & $2.108(1.219,3.645)$ & 0.008 \\
\hline$\geq 134$ & 429 & $3.191(1.862,5.468)$ & $<0.001$ \\
\hline SBP $(\mathrm{mmHg})$ & & & 0.458 \\
\hline$<114$ & 336 & $2.754(1.612,4.704)$ & $<0.001$ \\
\hline$\geq 114$ & 555 & $1.964(1.123,3.434)$ & 0.018 \\
\hline $\mathrm{DBP}(\mathrm{mmHg})$ & & & 0.343 \\
\hline$<60$ & 358 & $2.251(1.397,3.627)$ & 0.001 \\
\hline$\geq 60$ & 533 & $3.290(1.759,6.150)$ & $<0.001$ \\
\hline Heart rate (beats/min) & & & 0.935 \\
\hline$<87$ & 329 & $2.623(1.432,4.802)$ & 0.002 \\
\hline$\geq 87$ & 562 & $2.763(1.692,4.514)$ & $<0.001$ \\
\hline Respiratory rate (beats/min) & & & 0.524 \\
\hline$<19$ & 345 & $3.100(1.346,7.144)$ & 0.008 \\
\hline$\geq 19$ & 546 & $2.406(1.570,3.687)$ & $<0.001$ \\
\hline Temperature $\left({ }^{\circ} \mathrm{C}\right)$ & & & 0.977 \\
\hline$<36.8$ & 351 & $2.494(1.402,4.438)$ & 0.002 \\
\hline$\geq 36.8$ & 540 & $2.462(1.480,4.093)$ & 0.001 \\
\hline SPO2 (\%) & & & 0.212 \\
\hline$<97$ & 451 & $3.373(2.093,5.436)$ & $<0.001$ \\
\hline$\geq 97$ & 440 & $2.004(1.075,3.735)$ & 0.029 \\
\hline SOFA (score) & & & 0.947 \\
\hline$<5$ & 448 & $2.318(1.054,5.100)$ & 0.037 \\
\hline$\geq 5$ & 443 & $2.354(1.513,3.662)$ & $<0.001$ \\
\hline SAPSII score & & & 0.858 \\
\hline$<39$ & 539 & $2.303(1.145,4.634)$ & 0.019 \\
\hline$\geq 39$ & 352 & $2.183(1.362,3.501)$ & 0.001 \\
\hline Hyperensort use & & & 0.234 \\
\hline Yes & 673 & $2.725(1.581,3,697)$ & $<0.001$ \\
\hline No & 218 & $1.692(0.972,2.946)$ & 0.063 \\
\hline
\end{tabular}




\begin{tabular}{|lcccc|}
\hline Ventdurate & & & 0.742 \\
\hline Yes & 442 & $2.464(1.596,3.802)$ & $<0.001$ & \\
\hline Diabetes & 449 & $3.302(1.469,7.424)$ & 0.004 & \\
Yes & & & & 0.551 \\
\hline No & 223 & $3.344(1.627,6.872)$ & 0.001 & \\
AKI & 668 & $2,443(1,563,3.817)$ & $<0.001$ & \\
Yes & & & & 0.302 \\
\hline No & 167 & $1.930(1.043,3.571)$ & 0.036 & \\
\hline
\end{tabular}

SOFA: sequential organ failure assessment; SAPSII: simplified acute physiology score II.AKI:act kidney injury.

Figures 


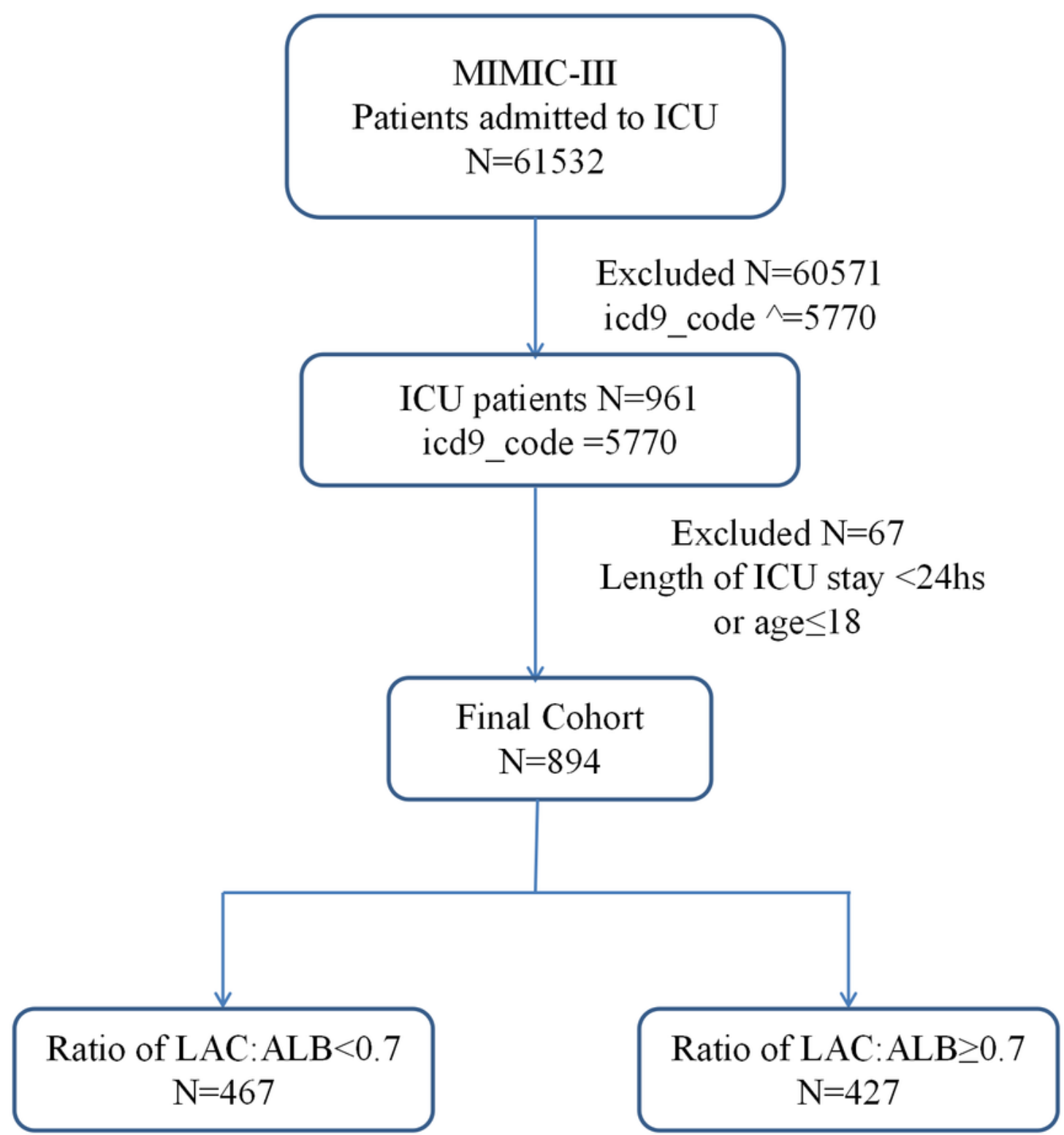

Fig.1 The detailed process of data extraction

Figure 1

See image above for figure legend 


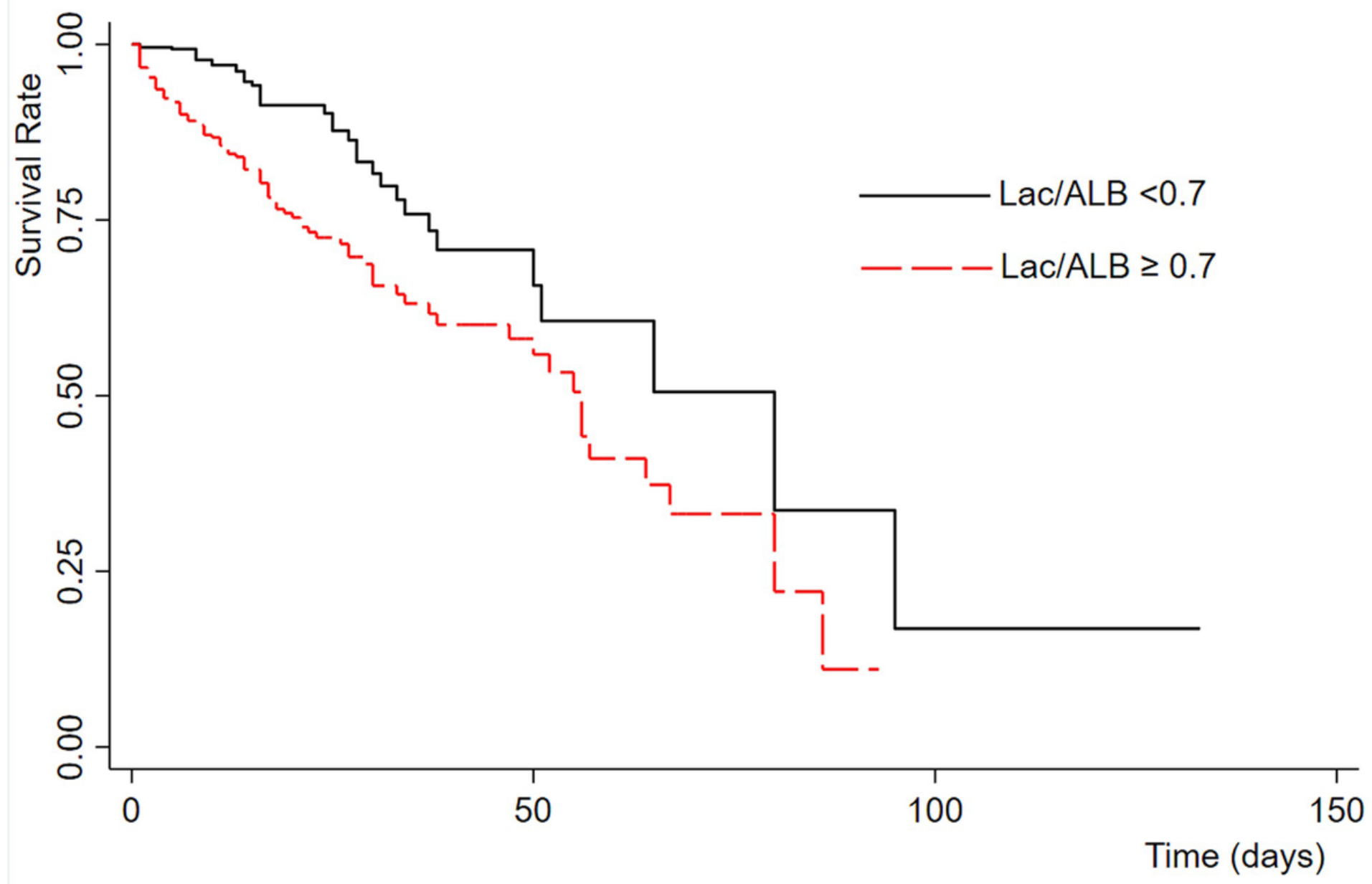

Figure. 2 Lac/ALB probability curves for mortality in acute pancreatitis. Median survival and $95 \mathrm{Cl}$ were 80 $(50,95)$ days in $L A C / A L B<0.7$ and $56(47,67)$ days in $\geq 0.7$, respectively. Log rank test $x 2=27.61, P<0.001$

Figure 2

See image above for figure legend 